\title{
Research on Single-phase Replacement of 500 kV Transformer based on PSCAD
}

\author{
LIU Mosi', a, SUN Zhiyuan"1, b \\ ${ }^{1}$ Electric Power Research Institute, Guangxi Power Grid Co. Ltd., Nanning, China \\ aliu_ms.sy@gx.csg.cn, bsun_zy.sy@gx.csg.cn,
}

Keywords: Transformer, Parameter inconsistency, Steady-state characteristics, Transient characteristics.

Abstract. In order to reduce outage time of the transformer after the single-phase fault, the fault phase is considered by using the spare device with similar parameters. However, the use of single-phase transformers with similar parameters to replace can lead some problems of operation because of the different parameters of the transformer. In this paper, the steady and transient problems encountered in the operation of single-phase replacement of transformer are studied based on PSCAD, and the feasibility of single-phase transformer replacement is discussed.

\section{Introduction}

As it takes too long for the transformer to repair, it will bring the hidden danger to the safety and stability of the power grid. If the same type of standby single-phase transformer can be used to replace the fault phase, the transformer outage time can be shortened.

Because the type of the spare transformer is different from the original transformer, the parameters may differ from the original transformer. If the single phase is replaced, the influence on the transformer itself and the system must be studied.

Taking a transformer in a $500 \mathrm{kV}$ substation which needs overhaul as an example, this paper discusses the single-phase substitution of the transformer of the $500 \mathrm{kV}$ station with the adjacent $500 \mathrm{kV}$ station. Considering the key indexes under the normal operation, the influence of the fault conditions on the protection and safety and stability of the control device based on PSCAD.

\section{Parameter Analysis about Single-phase Replacement of Transformer}

The simplified circuit of three-phase transformer with phase A replacement is shown in Fig. 1. Because of the inconsistency of parameters due to the replacement phase, the transformer will be operating asymmetrically, so it is necessary to study the influence on the transformer itself and the system.

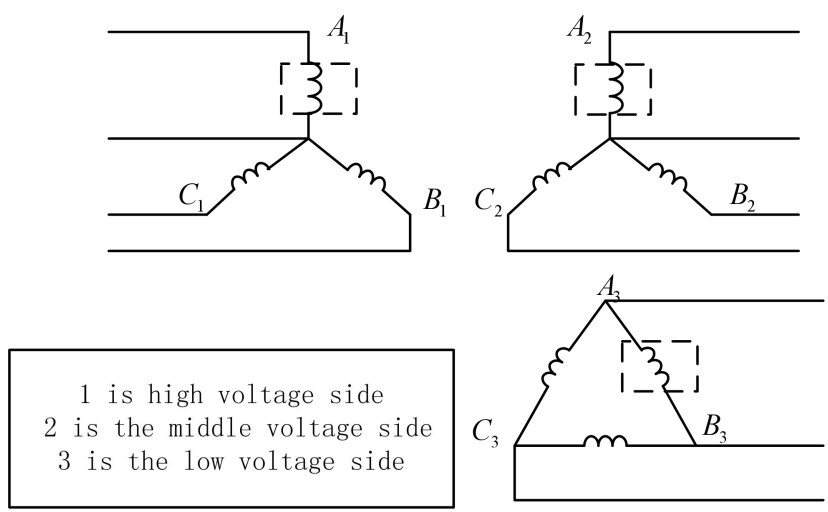

Fig. 1 simplified circuit of phase A replacement for three-phase autotransformer

The transformer single-phase replacement mainly changes the parameters of one phase, so that the voltage and current relationship is different from the original transformer. 


\section{Analysis of Operation Condition}

Operation Condition of Transformer. The transformer has the following provisions, the main parameters, $500 \mathrm{kV}$ transformer rated capacity, voltage combination, tapping range, connection, no-load loss and load loss and no-load current and short-circuit impedance should comply with the requirements. The operating voltage of the transformer shall not be higher than $105 \%$ of the tap voltage of the operation, and shall not exceed the maximum operating voltage of the system [1].

Parallel Operation Condition of Transformer. The parallel operation of the transformer shall meet the following requirements, the same value of the connection group, the voltage ratio shall be the same, which the difference shall not exceed $0.5 \%$, the value of the impedance voltage shall not exceed $10 \%$ [2].

System Voltage and Current Requirements. When normal operation of power grid, the negative sequence of voltage shall not exceed $2 \%$, and not exceed $4 \%$ within a short time. Each user connected to the common connection point causes the negative sequence of voltage below $1.3 \%$, and no more than $2.6 \%$ within a short time [3].

Excessive negative sequence current will cause overheating of the motor in the system. When the negative sequence current does not exceed $5 \%$ of the positive sequence current, the three-phase current is actually balanced $[4,5]$.

The voltage and current unbalance degree is expressed as shown in Eq. 1 and Eq. 2.

$$
\begin{gathered}
\partial U \%=\frac{\left|U_{2}\right|}{\left|U_{1}\right|} \\
\partial I \%=\frac{\left|I_{2}\right|}{\left|I_{1}\right|}
\end{gathered}
$$

Wherein, $U_{1}$ indicates positive sequence voltage, $U_{2}$ indicates negative sequence voltage, $I_{1}$ indicates positive sequence current, $I_{2}$ indicates negative sequence current, $\partial U \%$ and $\partial I \%$ indicate voltage and current unbalance respectively.

Fault State Characteristic Analysis. In this paper, PSACD is used to simulate the transient voltage and current, and analyze whether the change of electrical quantity in short circuit fault can meet the original protection and safety stability setting.

\section{Simulation Analysis based on PSACD}

Taking the replacement of phase A of transformer in a $500 \mathrm{kV}$ station as an example, the PSCAD simulation analysis is carried out to discuss the single-phase replacement parameters and operating conditions.

According to the power flow distribution of the area, PSD-BPA software is used for power flow calculation, which get the equivalent impedance of $500 \mathrm{kV}$ side of the transformer, equivalent load of the $220 \mathrm{kV}$ side. And then equivalent system is established using PSCAD with these parameters.

Steady State Operation Analysis. According to the no-load, normal load and full load conditions, the voltage and current imbalance degree of the three side of the transformer is simulated and analyzed, and the results are shown in Table 1 and Table 2. 
Table 1 Negative sequence unbalance of voltage at three sides of transformer before and after replacement

Negative sequence unbalance of voltage [\%]

Load condition

Primary transformer with no-load

Primary transformer with normal load

Primary transformer with full-load

Replaced with no-load

Replaced with normal load

Replaced with full-load
High voltage side

$4.33 \mathrm{E}-08$
$4.26 \mathrm{E}-07$
$4.26 \mathrm{E}-06$
$3.22 \mathrm{E}-05$
$8.73 \mathrm{E}-05$
$6.32 \mathrm{E}-04$

Medium voltage side

4.33E-08

4.33E-08

$1.17 \mathrm{E}-06$

$2.96 \mathrm{E}-02$

$3.16 \mathrm{E}-02$

7.18E-02
Low voltage side

4.33E-08

$1.32 \mathrm{E}-06$

$1.32 \mathrm{E}-06$

2.72E-02

4.23E-02

$1.60 \mathrm{E}-01$

According to the simulation results of the phase A before and after replacement of the transformer, it can be seen that with the increase load of the medium voltage side, the imbalance of the negative sequence of voltage at the three side of the transformer is increased. Among them, the increment of unbalance is more obvious after phase A replacement. The unbalance of negative sequence in the middle and low voltage side is more than that of the high voltage side after the replacement, and the imbalance of negative sequence at the low voltage side is the most. The simulation results show that the unbalance of the high voltage side, the medium voltage side and the low voltage side before and after the replacement are less than $0.2 \%$ under the three load conditions.

Table 2 Negative sequence unbalance of current at three sides of transformer before and after replacement

Negative sequence unbalance of current [\%]

Load condition

Primary transformer with no-load

Primary transformer with normal load

Primary transformer with full-load

Replaced with no-load

Replaced with normal load

Replaced with full-load
High voltage side

4.78E-04

6.71E-08

5.37E-08

2.72E-02

4.99E-02

8.97E-02
Medium voltage side

4.61E-06

1.57E-07

$3.52 \mathrm{E}-07$

7.65E-06

$3.16 \mathrm{E}-02$

7.22E-02
Low voltage side

4.78E-04

1.69E-07

$1.22 \mathrm{E}-07$

2.77E-02

4.27E-02

$1.60 \mathrm{E}-01$

With the increase of the load of medium voltage side, the negative sequence imbalance of current of the three side is increased, and the current imbalance is larger than the voltage imbalance before and after the replacement of the phase A. The simulation results show that the negative sequence imbalance of current of the high voltage side, the medium voltage side and the low voltage side before and after replacement are less than $0.2 \%$.

Transient Analysis. When the single-phase short circuit occurs at the outlet of the main voltage side of the transformer, the short circuit current and the phase voltage before and after the replacement are shown in Fig. 2 and Fig. 3.

When the single-phase short circuit occurs at the outlet of the main voltage side of the transformer, The RMS of short-circuit current after replacement is increase by $1.2 \mathrm{kA}$. After replacement, the short circuit current and voltage waveform of phase A is basically the same as before. 


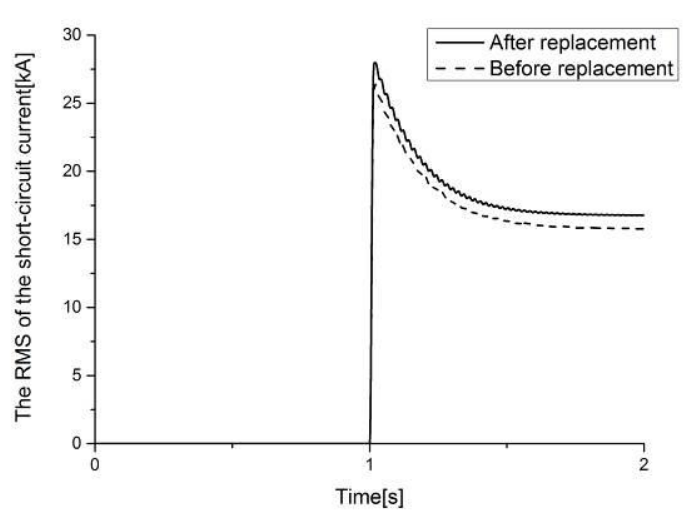

Fig. 2 Short-circuit current curve

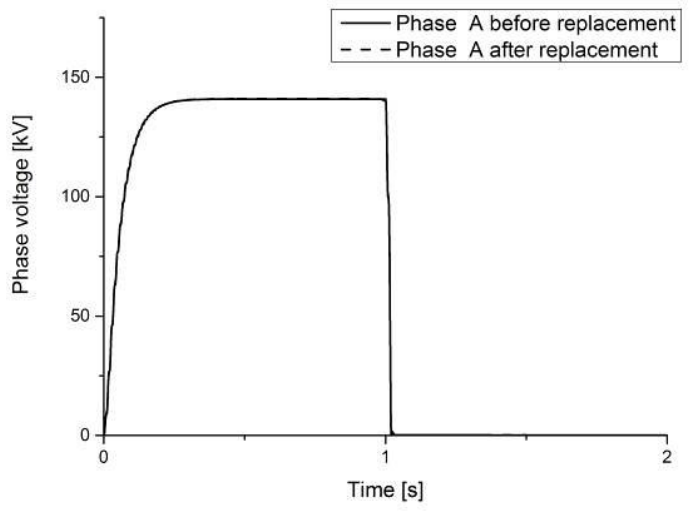

Fig. 3 Phase voltage curve

When the interphase short circuit occurs at the outlet of the middle voltage side of the transformer, the short-circuit current and the phase voltage before and after the replacement are shown in Fig. 4 and Fig. 5.

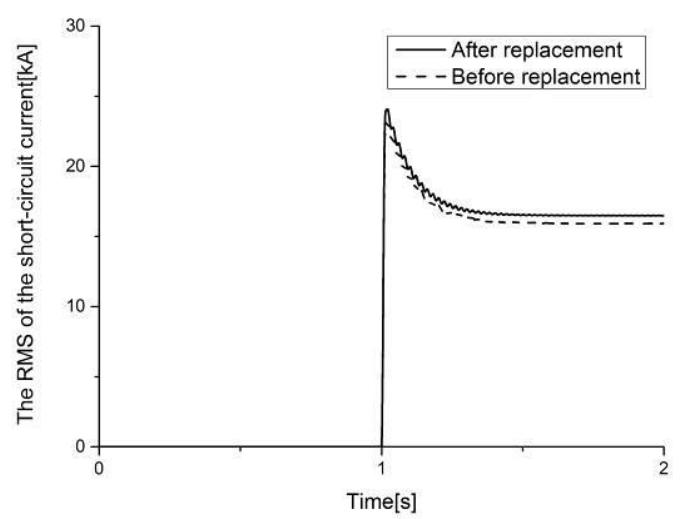

Fig. 4 Short-circuit current curve

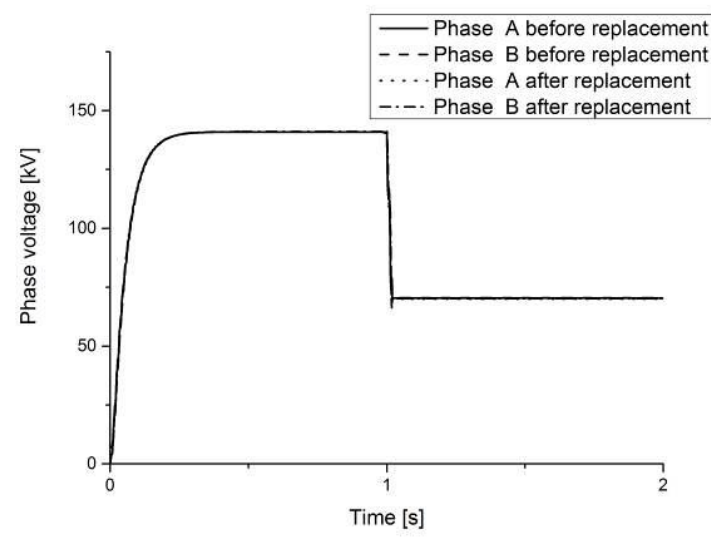

Fig. 5 Phase voltage curve

When the Interphase short circuit occurs at the outlet of the middle voltage side of the transformer, the RMS of short-circuit current after replacement is increase by $0.6 \mathrm{kA}$. After replacement, the short circuit current and voltage waveform of phase $\mathrm{A}$ is basically the same as before.

Through the simulation for the single-phase short circuit and Interphase short circuit, it is found that the short circuit current increases after replacement, and the waveform is basically the same as before.

\section{Conclusions}

In the steady state operation, the main parameters and operation voltage should meet the requirements of the regulations. The negative sequence unbalance of voltage and current of the transformer should meet the operation requirements after replacement. When the system is short circuited, it is necessary to study whether the change of voltage and current meets the original protection setting after replacement.

Taking the $500 \mathrm{kV}$ transformer replacement of a substation as an example, the voltage and current unbalance of the three side of the transformer are simulated and analyzed under three load conditions. The results show that the negative sequence unbalance of voltage and current are less than $0.2 \%$ after single-phase replacement. In the form of two kinds of short circuit, the short-circuit current is slightly larger than before, and does not affect the relay protection settings. 


\section{References}

[1] GB/T 6451-2015 Specification and technical requirements for oil-immersed power transformers

[2] DL/T 572-2010 Operation specification for power transformer

[3] GB/T 15543-2008 Power quality_—Three-phase voltage unbalance

[4] ZHANG Diansheng: Handbook of power transmission line design for Power Engineering (China Electric Power Press, China 2002).

[5] X Chengjun, H Dongyan, D Wenliang and Q Guihua: Feasibility analysis of single phase replacement for $500 \mathrm{kV}$ main transformers. Power System Protection and Control. Vol. 39(2011), p. 93-97. 